\title{
Multi-objective Human-computer Co-operative Co-evolutionary Method Based on Non-dominated Sorting Strategy
}

\author{
Huo Junzhou ${ }^{1,3}$, Chen Jing ${ }^{2, *}$ Zhou Jianjun $^{3}$ and Wu Hanyang ${ }^{1}$ \\ ${ }^{I}$ Dalian University of Technology, Dalian 116024, P.R. China \\ ${ }^{2}$ Dalian Ocean University, Dalian 116023, P.R. China \\ ${ }^{3}$ State Key Laboratory of Shield Machine and Boring Technology, Zhengzhou 450001, China
}

\begin{abstract}
Based on the human-computer cooperation ideas, a Human-Computer Multi-Objective Cooperative Co-Evolutionary Method (HCMCCM) is developed to solve the complex engineering layout problem, in which the multiobjective optimization idea is integrated to avoid the "flooding" phenomenon that occurs during the combination of the artificial solutions and the algorithm solutions. In the proposed HCMCCM, the artificial solutions expressed by unified encoding strings are incorporated together with the algorithms solutions to create new cooperative solutions based on the non-dominated sorting strategies. This kind of cooperation can make the artificial solutions and the algorithm solutions on an equal basis and integrate the artificial individual with the individual algorithms into a multi-objective trade-off. The numerical simulation results of the satellite layout problem show that the proposed method can combine the artificial solutions and the algorithm solutions effectively and provide a Pareto solution set for engineers to choose from.
\end{abstract}

Keywords: Co-evolutionary, human-computer cooperation, multiobjective, spatial layout.

\section{INTRODUCTION}

Multiobjective layout design problems exist in various engineering fields. Different layout problems usually have different theoretical basis and different level of engineering complexity. It is difficult to give a general definition of the complex layout problem. Dowsland [1], Michalek [2] and Cagan [3] pointed out that the spatial layout problem concerns the placement of components in an available space such that a set of objectives can be optimized while satisfying optional spatial or performance constraints.

In order to solve the complex multi-objective layout problem more effectively, many scholars have been studying the human-machine integration method, in which engineers playing in the visual, thinking in images, macro-controlling and other aspects of expertise are used to deal with the complex human-machine layout problems. Based on efficient solving algorithms, Cho [4] proposed an interactive genetic algorithm, where human can evaluate solutions' fitness values; Kosorukoff [5] presented a human based genetic algorithm, where human not only can evaluate solutions' fitness values but also control the genetic operations. Liu and Teng [6] presented a human-algorithm-knowledge based human-computer cooperative layout method to solve a satellite module layout problem. In addition, Murthy [7] proposed an asynchronous team framework which substituted the algorithm operators.
Researches on the evolutionary algorithm-based humancomputer co-operation have made encouraging progress.

In summary, the above-mentioned integration often results in a flooding phenomenon between the artificial solutions and the human solutions. The number of artificial solutions online obtained by a priori knowledge is very limited. During the combination of the human solutions and the algorithm solutions, the multi-objective optimization problem is often transferred into a single objective problem by the weighted sum approach. So if the artificial solutions were directly added into the algorithms population, two extreme circumstances may probably occur: one is that the artificial solutions are "drowned" in the algorithms' population and will not achieve their guiding effect because the number of artificial solutions is too small. Another one is that the best solution of the algorithm is replaced by the artificial solutions and makes the algorithm fall into a local search area because the artificial solution is too good and the potential of the algorithm has not been fully explored. So the later cooperation way essentially plays an "alternative" role instead of "integration" role.

Based on the above-mentioned analysis, in this study, a multi-objective non-dominated sorting strategy is used to incorporate the artificial solutions and the algorithm solutions into a new solution set, where the artificial solutions are expressed by unified encoding strings. The proposed method is used to solve a satellite layout problem with multiple engineering constraints to demonstrate its feasibility and effectiveness. 


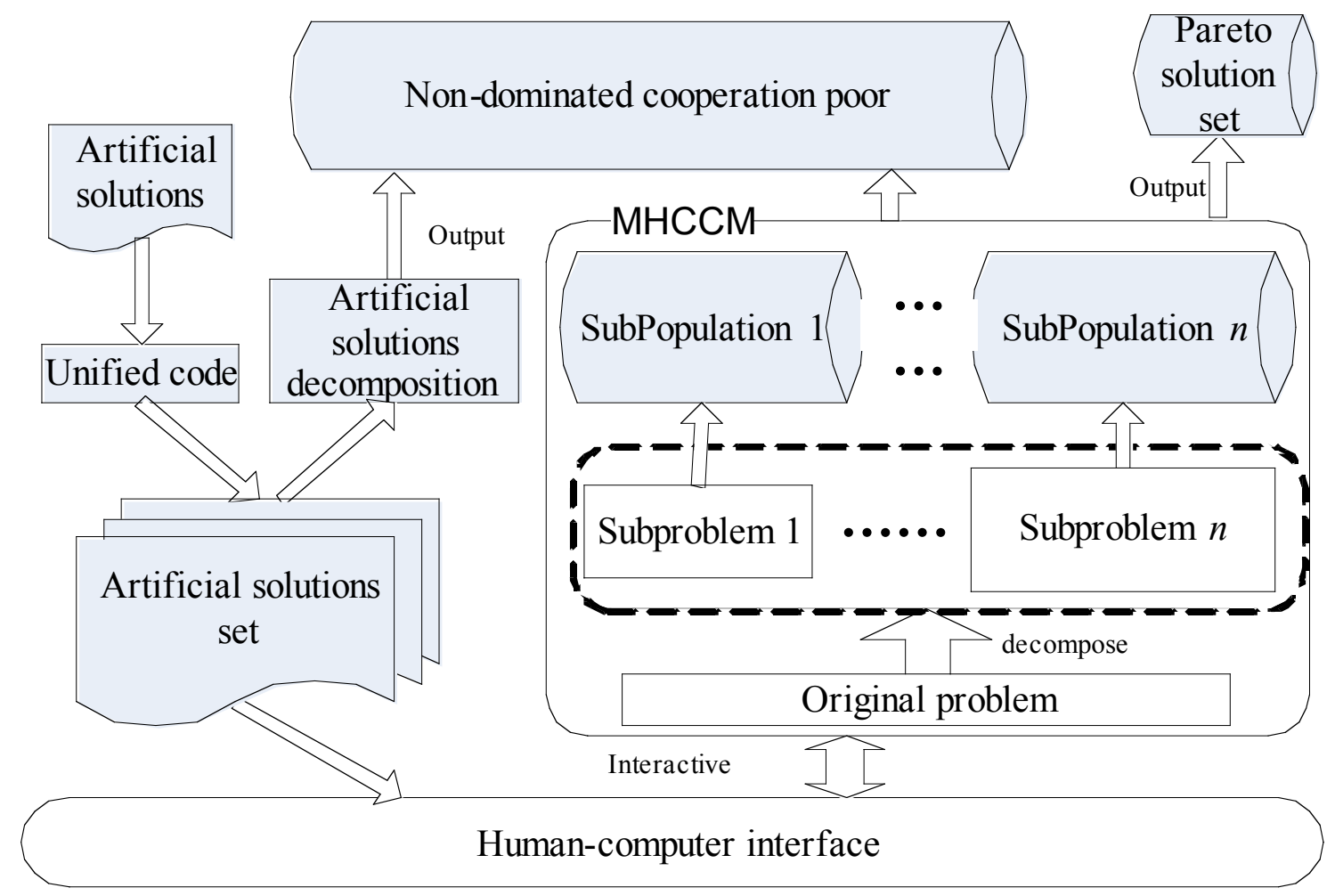

Fig. (1). Framework of the HCMCCM.

\section{HUMAN-COMPUTER MULTIOBJECTIVE CO- EVOLUTIONARY METHOD}

\subsection{The Whole Framework}

The framework of the HCMCCM was shown in Fig. (1).

\subsection{Multiobjective Cooperative Co-evolutionary Algo- rithm}

Recently the Multi-objective Co-Evolutionary Algorithm MOCEA has been widely studied. Keer attempted to extend multi-objective optimization algorithm into the CCEA framework, in which every variable was set by a population and the framework proposed by Fonseca and Fleming [8] was adopted. Coello [9] introduced some definition of the CCEA into multi-objective optimization and proposed a collaborative multi-objective method. Tan [10] proposed a multiobjective cooperative co-evolutionary algorithm.

It can be seen that the present studies on MOCEA mainly involve the decomposition of the problem into sub-problems and the integration of the multi-objective searching strategies with the CCEA. In this study, drawing on the multi-objective optimization technique, the non-dominated sorting strategies and cooperative co-evolutionary algorithms are combined to solve the complex layout optimization problem. First, the original problem is decomposed into several sub-problems according to the characteristics of the problem. Then, each sub-problem is assigned a population. Finally, the nondominated sorting and the crowding out mechanisms proposed by Deb [12] are used to rank the individuals and update Pareto set.

\subsection{The Integration of the Artificial Solutions and the Algorithm Solutions}

It was stated earlier that there were two integration ways; the replacement way and the cross way. These two kinds of integration ways cannot achieve a sustainable effect because an'unfair'integration often takes place. This study focuses on studying the integration among the human experience, the multiobjective strategies and the CCEA, in which the integration can make the artificial solutions and algorithm solutions combined on an equal basis and realize the integration in multi-objective trade-off instead of simply adding the artificial solutions into the algorithm population and fend for them.

\section{NUMERICAL EXPERIMENTS}

Suppose that there are forty five objects that need to be located on two bearing surfaces in a satellite module. The dimensions and the masses of the module can be found in reference [11]. The goal of the problem is to optimize the mass distribution of the whole system, the gathering capability, the disturbing capability and the accessibility directions until many complex technical constraints are satisfied. Two methods were used to solve the satellite layout problem and each of them randomly ran 30 times. The two methods were a Cooperative Co-Evolutionary Genetic Algorithm (CCGA) and the proposed HCMCCM.

The comparison of the statistic data of the two methods are shown in (Table 1). The layout patterns obtained by the CCGA and the HCMCCM are illustrated as Fig. (2). The fitness value profiles and the diversity distribution curve of 


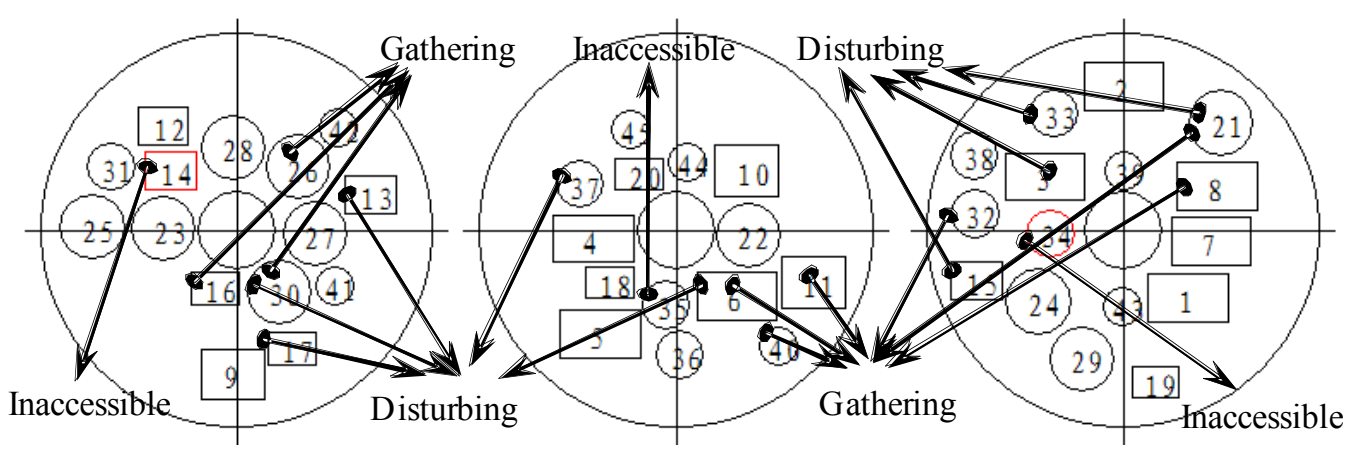

The best layout pattern obtained by the CCGA

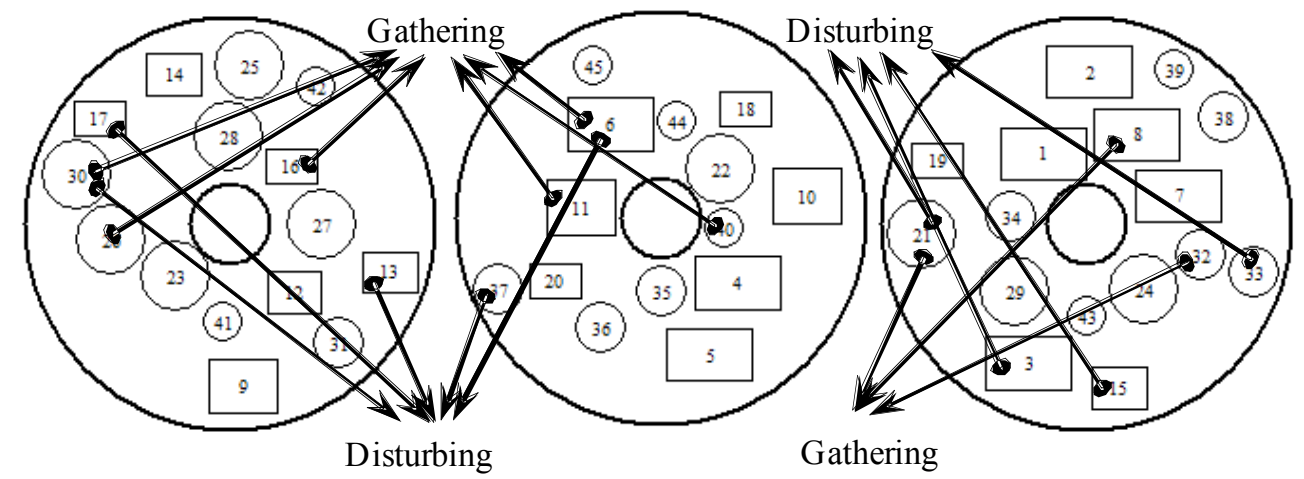

The best layout pattern obtained by the HCMCCM

Fig. (2). The layout patterns obtained by the CCGA and the HCMCCM.

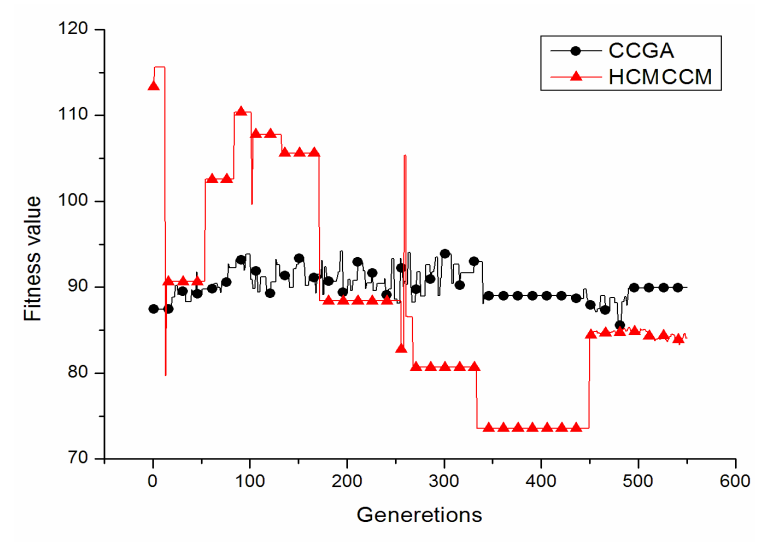

(a) The fitness value profiles

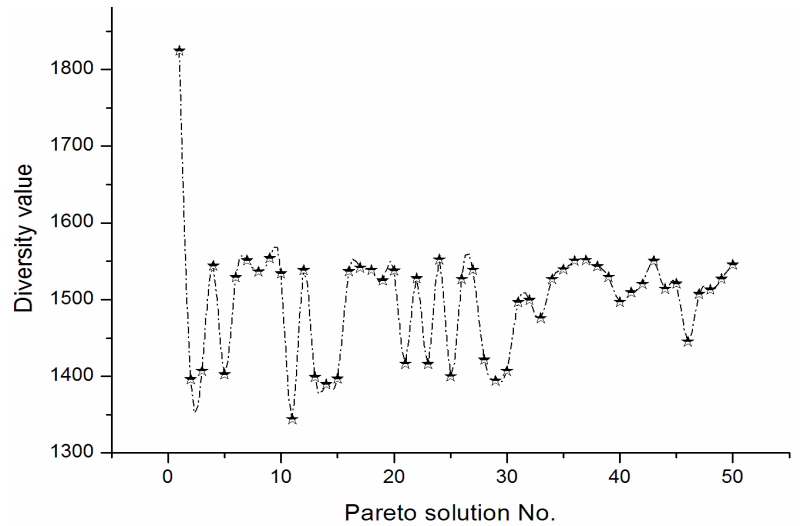

(b) Pareto solutions differences obtained by HCMCCM

Fig. (3). Fitness value profiles and the diversity distribution curve of the two methods.

the two methods are illustrated as Fig. (3). Each set of data in (Table 1) denotes the mean value, standard deviation value, minimum and maximum value of index, respectively.

Comparing the HCMCCM and the CCGA on the objectives, the constraints, the success probability, and the runtime (Table 1), it can be observed that:

- The HCMCCM keeps a higher success probability.

- The sum of the lowest value and the average value of the inertia obtained by the proposed HCMCCM are higher than those of the CCGA.
- The best value and the average value of the gathering weight obtained by the HCMCCM are lower than those of the CCGA.

- The best value and the average value of the disturbing weight obtained by the HCMCCM are highest. It means that the optimal layout scheme obtained by HCMCCM can make all disturbing objects further away from each other.

- The HCMCCM can produce the best solutions with the highest accessibility directions that can make the objects easy to be modify or assemble. 
Table 1 The comparison of the statistic data of the two methods.

\begin{tabular}{|c|c|c|c|}
\hline \multicolumn{2}{|c|}{ Methods } & \multirow{2}{*}{$\frac{\text { CCGA }}{(1125.3,73.5,1111.2,1132.8)}$} & \multirow{2}{*}{$\frac{\text { НСМССМ }}{(1131.4,113.5,1121.4,1138)}$} \\
\hline \multirow{3}{*}{ In the folded solar panel } & $I_{x}+I_{y}+I_{z}\left(\mathrm{Kg} \cdot \mathrm{m}^{2}\right)$ & & \\
\hline & $\left|\theta_{x}\right|+\left|\theta_{y}\right|+\left|\theta_{z}\right|(\mathrm{rad})$ & $(0.006,0.036,0.001,0.018)$ & $(0.01,0.086,0.005,0.027)$ \\
\hline & $\left|\delta x_{c}\right|+\left|\delta y_{c}\right|(\mathrm{mm})$ & $(0.27,8.1,0.000,6.3)$ & $(1.5,9.8,0.01,2.9)$ \\
\hline \multirow[t]{2}{*}{ In the unfolded solar panel } & $\left|\theta_{x}\right|+\left|\theta_{y}\right|+\left|\theta_{z}\right|(\mathrm{rad})$ & $(0.004,0.022,0.001,0.013)$ & $(0.02,0.123,0.008,0.026)$ \\
\hline & $\left|\delta x_{c}\right|+\left|\delta y_{c}\right|(\mathrm{mm})$ & $(0.16,5.35,0.000,3.5)$ & $(1.4,9.67,0.1,2.6)$ \\
\hline \multicolumn{2}{|c|}{ The success probability (\%) } & 85 & 95 \\
\hline \multicolumn{2}{|c|}{ Object area beyond the fixed area } & $(2.6,29.8,0,9.9)$ & $(1.7,24.5,0,10.5)$ \\
\hline \multicolumn{2}{|c|}{ The gathering value } & $(22421,3268,21534,23737)$ & $(20234,5462,19856,25645)$ \\
\hline \multicolumn{2}{|c|}{ The disturbing value } & $(26568,9604,24352,28444)$ & $(29845,3476,25467,31456)$ \\
\hline \multicolumn{2}{|c|}{ The accessibility directions } & $(43.68,3.2,43,44)$ & $(45,0,45,45)$ \\
\hline
\end{tabular}

- The proposed HCMCCM needs the longest runtime and CCGA has the shortest runtime.

As shown in Fig. (2), the word "grouping" means the locations of the layout objects are expected to be the nearer the better; the word "disturbing" means the locations of the layout objects that are expected to be the father away from each other the better and the word "inaccessible" denotes the layout objects that have no accessibility to assemble or modify. Comparing the above-mentioned two best layout patterns, the best layout pattern obtained by the HCMCCM can tackle some objectives or constraints which cannot be formulated precisely. For example, for the accessibility direction requirements, the HCMCCM can obtain many good schemes in which the objects' layout pattern is easy to be modified or assembled.

As shown in Fig. (3), during the optimization process, the best fit curve of HCMCCM method is constantly oscillating, it can be seen that the proposed method can integrate the artificial solutions with the algorithm solutions continuously.

\section{CONCLUSION}

To avoid the "flooding" phenomenon during the integration of artificial solutions with the algorithm solutions, this paper adopts the non-dominated sorting strategy to integrate the artificial solutions with the algorithm solutions, which can make the integration process more effective on the basis of equality. The results show that the proposed method can add the artificial solutions into the algorithm's population efficiently without being "drowned". It can achieve higher levels of sustainability and effectiveness with the equal combination of the two kinds of solutions. It can make full use of the human experience and the algorithm to solve the complex engineering problems more effectively.

\section{CONFLICT OF INTEREST}

The authors confirm that this article content has no conflict of interest.

\section{ACKNOWLEDGEMENTS}

This project is supported by the National Natural Science Foundation of China (Grant No. 51375001and No. 51309045), and the Program for Liaoning Excellent Talents in University (Grant No. LJQ2014075).

\section{REFERENCES}

[1] W. B. Dowsland, "Three-dimensional packing-solution approaches and heuristic development," Int. J. Prod. Res., vol. 29, no. 8, pp. 1673-1685, 1991.

[2] R. S. Liggett, and W. J. Mitchell, "Optimal Space Planning in Practice”, Comput. Aided. Des., vol.13, p. 277, 1981.

[3] J. Cagan, K. Shimada, and S. Yin, "A survey of computational approaches to three-dimensional layout problems", Comput. Aided. Des., vol. 34, p. 597, 2002.

[4] S.B. Cho, "Towards creative evolutionary systems with interactive genetic algorithm,". Appl. Intell., vol. 16, no. 2, pp. 129-138, Mar. 2002.

[5] C. D. Cheng, and A. Kosorukoff, "Interactive One-Max Problem Allows to Compare the Performance of Interactive and HumanBased Genetic Algorithms, "LNCS., vol. 3102, pp. 983-993, 2004. 
[6] Z. W. Liu, and H. F Teng, "Human-computer cooperative lay- out design method and its application", Comput. Ind. Eng., vol. 55, pp. 735-757, 2008.

[7] J. Rachlin, R. Goodwin, S. Murthy, R. Akkiraju, F. Wu, S. Kumaran, and R. Das, "A-Teams: An agent architecture for optimization and decision-support", LNCS., vol. 1555, p. 261, 1999.

[8] C. M. Fonseca, and P. J. Fleming, “An overview of evolutionary algorithms in multiobjective optimization," Evolut. Comput., vol. 3, pp. 1-16, 1995.
[9] M. Reyes-Sierra, and C. A. Coello Coello, "Multi-objective particle swarm optimizers: a survey of the state-of-the-art," Int. J. Comp., vol. 2, no. 3, pp. 287-308, 2006.

[10] K. C. Tan, Y. J. Yang, and C. K. Goh, "A distributed coorperative coevolutionary algorithm for multiobjective optimizatio", IEEE. T. Evolut. Comput., vol. 10, p. 527, 2006.

[11] J. Z. Huo, H. F. Teng, W. Sun, and J. Chen, "Human-computer cooperative co-evolutionary method and its application to a satellite module layout design", Aeronaut. J., vol. 114, p. 209, 2010.

[12] K. Deb, A. Pratap, S. Agarwal, and T. Meyarivan, "A fast and elitist multiobjective genetic algorithm: NSGA-II", IEEE. T. Evolut. Comput., vol. 6, pp. 182-197, 2002.

(C) Junzhou et al:; Licensee Bentham Open.

This is an open access article licensed under the terms of the Creative Commons Attribution Non-Commercial License (http://creativecommons.org/licenses/by-nc/3.0/) which permits unrestricted, non-commercial use, distribution and reproduction in any medium, provided the work is properly cited. 